\title{
El riesgo en el almacenamiento de GLP en el Ecuador
}

\section{The risk of LPG storage in Ecuador}

Diego Fernando Venegas Vásconez

Universidad de Concepción, Chile

Cesar Ricardo Ayabaca Sarria

Escuela Politécnica Nacional, Ecuador

Santiago Fernando Celi Ortega

Universidad Internacional SEK Ecuador, Ecuador

Juan Carlos Rocha Hoyos

Universidad Internacional SEK Ecuador, Ecuador

Autor para correspondencia: diegofvenegas@udec.cl, cesar.ayabaca@epn.edu.ec, santiago.celi@uisek.edu.ec, carlos.rocha@uisek.edu.ec

Fecha de recepción: 19 de Julio 2017 - Fecha de aceptación: 15 de Enero de 2018

Resumen: El gas licuado de petróleo (GLP) es un combustible que proporciona energía a equipos y maquinarias para su funcionamiento, y es utilizado en el Ecuador en aplicaciones residenciales, comerciales e industriales. El manejo y utilización de este combustible genera riesgos hacia las personas y edificaciones que se encuentran en sus cercanías, los cuales no pueden ser eliminados, pero si pueden ser minimizados, si se lo manipula de forma técnica.

El presente trabajo muestra algunos de los problemas de seguridad más recurrentes que existen en el almacenamiento del GLP. También se muestran algunas de las obligatoriedades establecidas en la Norma Técnica INEN 2260:2010 para las instalaciones de este combustible, y como son acatadas por los involucrados en la seguridad de estos sistemas para beneficio de la ciudadanía.

Palabras claves: gas licuado de petróleo; seguridad; instalación; norma técnica; almacenamiento

Abstract: Liquefied petroleum gas (LPG) is a fuel that provides energy to equipment and machinery for its operation, and is used in Ecuador in residential, commercial and industrial applications. The handling and use of this fuel creates risks to the people and buildings that are nearby, which can't be eliminated, but can be minimized if it is handled in a technical way.

The present work shows some of the most recurrent safety problems that exist in the storage of LPG. Also shown are some of the mandatory provisions established in Technical Standard INEN 2260: 2010 for the facilities of this fuel, and as they are accepted by those involved in the safety of these systems for the benefit of citizens.

Key words: liquefied petroleum gas; safety; installation; technical standard; storage 


\section{Introducción}

El gas licuado de petróleo (GLP) es un combustible muy utilizado como una fuente de energía, sobre todo por sus múltiples ventajas, entre las que se pueden mencionar:

- Alto poder calorífico comparado con otras fuentes de energía (Boggavarapu, Ray, \& Ravikrishna, 2014).

- Limpio en términos de emisiones de gases contaminantes (Raslavi, Mockus, Ker, \& Starevi, 2014).

- Se combustiona completo, por eso no produce hollín (Kim, Kim, \& Oh, 2016).

- Es fácil de transportarlo y almacenarlo por ello se lo licua, ya que a condiciones ambientales, el GLP se encuentra en fase vapor (Leporini, 2017).

- Su manipulación es muy segura si se lo maneja técnicamente (Venegas \& Ayabaca, 2017).

- $\quad$ No es tóxico para el ser humano (Chica, Espinoza, \& Rivera, 2010).

- Como una sola fuente de energía puede satisfacer varias necesidades energéticas (Kities, Mulder, \& Rietveld, 2014).

Sin embargo, a pesar de todas estas ventajas, se han detectado varios problemas que incrementan el riesgo por su manejo en las instalaciones cuando no se toman las precauciones debidas, entre las que se pueden mencionar:

- Desconocimiento de Normas Técnicas vigentes al momento de proyectar, diseñar, planificar, instalar y mantener sistemas de GLP, lo cual deriva en malas aplicaciones (Venegas, 2016).

- Falta de previsión en el diseño original de nuevas instalaciones para el espacio destinado al almacenamiento, por lo que los tanques están siendo instalados en sitios poco seguros (Venegas \& Farías, 2017).

- Falta de protección a los recipientes que contienen el combustible (Venegas Vásconez et al., 2017).

- Falta de seguimiento en el mantenimiento técnico de una instalación (Venegas et al., 2016a).

Todos estos problemas detectados han ocasionado múltiples accidentes, de los cuales, la BLEVE “Boiling Liquid Expanding Vapour Explosion”(Abbasi \& Abbasi, 2007), es el más peligroso y dañino (Planas, Pastor, Casal, \& Bonilla, 2015).

La BLEVE es un fenómeno de explosión de vapores que se expanden al hervir un líquido dentro de un recipiente a presión (International Labour Office, 1993). Se produce cuando un recipiente de almacenamiento del combustible es expuesto a fuego directo por un largo periodo de tiempo, se produce fatiga en la parte más débil del tanque, y ruptura (Figura 1), ocasionando una salida brusca del combustible que está en su interior (líquido y vapor). Se produce una expansión volumétrica del líquido en relación de 273/1 (Pérez Carmona, 2001) y al existir fuego exterior, este combustiona, avivando más el fuego y liberando energía con desprendimiento de fragmentos del tanque (Eckhoff, 2014). 


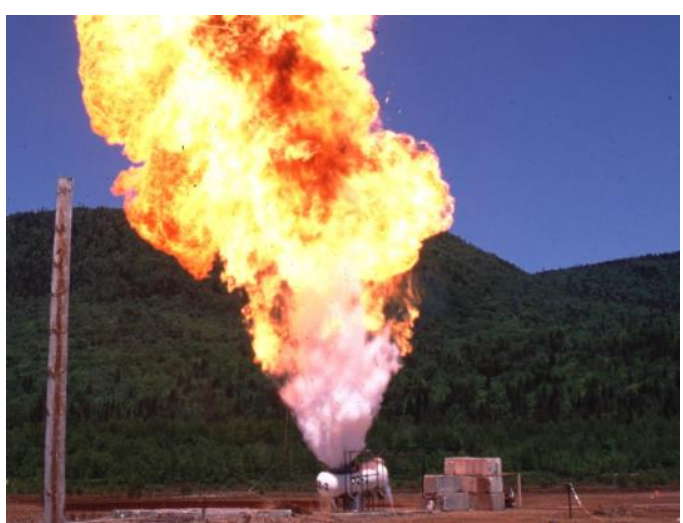

Figura 1. BLEVE en tanque de GLP (Nardini \& Nolasco, 2014)

El peligro que conlleva la BLEVE está relacionado con la destrucción total que ocasiona en todo lo que encuentra a su paso (Shariff, Wahab, \& Rusli, 2016). 1:

Hay varias causas por las que se produce este fenómeno, las cuales se detallan en la Tabla

Tabla 1: Causas más comunes para que ocurra una BLEVE (Prugh, 1991)

\begin{tabular}{lc} 
Causas de BLEVE & $\%$ \\
\hline Fuego & 26 \\
Rebosamiento & 18 \\
Descarrilamiento & 20 \\
Reacción fuera de control & 12 \\
Colisión & 10 \\
Sobrepresión & 6 \\
Otros & 8 \\
Total & 100 \\
\hline
\end{tabular}

Fuente: Elaboración propia

Existen reportes de múltiples incidentes a nivel mundial con BLEVE (Abbasi \& Abbasi, 2008), los cuales han generado conciencia en los organismos de prevención y control, que tratan por todas las formas de minimizar la ocurrencia de BLEVE en los sistemas de GLP, de lo cual el Ecuador no es la excepción y para ello el Instituto Ecuatoriano de Normalización ha elaborado la Norma Técnica INEN 2260:2010 (INEN Ecuador, 2010), la cual establece los parámetros mínimos de seguridad que deben tener las instalaciones de este combustible.

Siendo el manejo del GLP en fase líquida la parte más peligrosa de una instalación, con respecto a los recipientes de almacenamiento la Norma Técnica obliga a alejarlos de posibles puntos de inflamación o agentes que en un momento determinado puedan incrementar un incidente con presencia de fuego. En la Tabla 2 se indican las distancias mínimas de seguridad a terceros que dependen del volumen de almacenamiento y de la ubicación del recipiente (sobre o bajo superficie). 
Tabla 2: Distancias de seguridad de recipientes a terceros (INEN Ecuador, 2010)

\begin{tabular}{|c|c|c|c|c|c|c|c|c|c|c|c|c|c|c|c|c|c|c|c|}
\hline \multirow[t]{2}{*}{$\begin{array}{l}\text { Clasifi- } \\
\text { cación }\end{array}$} & \multicolumn{14}{|c|}{ INSTALACIONES SOBRE NIVEL DEL TERRENO } & \multicolumn{5}{|c|}{$\begin{array}{l}\text { INSTALACIONES } \\
\text { ENTERRADAS }\end{array}$} \\
\hline & \multicolumn{2}{|c|}{ A-A } & \multicolumn{2}{|c|}{$\mathrm{A}-\mathrm{O}$} & \multicolumn{2}{|c|}{ A-1 } & \multicolumn{2}{|c|}{$A-2$} & \multicolumn{2}{|c|}{ A-3 } & \multicolumn{2}{|c|}{$A-4$} & \multicolumn{2}{|c|}{ A-5 } & $E-E$ & $E-0$ & $E-1$ & $E-2$ & $E-3$ \\
\hline Volúmen $V \mathrm{~m}^{3}$ & \multicolumn{2}{|c|}{$0,11<V \leq 1$} & \multicolumn{2}{|c|}{$1<V \leq 5$} & \multicolumn{2}{|c|}{$5<\mathrm{V} \leq 10$} & \multicolumn{2}{|c|}{$10<V \leq 20$} & \multicolumn{2}{|c|}{$20<V \leq 100$} & \multicolumn{2}{|c|}{$100<V \leq 500$} & \multicolumn{2}{|c|}{$500<V \leq 2000$} & \multirow{2}{*}{$\begin{array}{c}0,11< \\
\mathrm{V} \leq 1\end{array}$} & \multirow[t]{2}{*}{$1<\mathrm{V} \leq 5$} & \multirow[t]{2}{*}{$5 \times \sqrt{ } \leq 10$} & \multirow{2}{*}{$\begin{array}{l}10<\mathrm{V} \\
\leq 100\end{array}$} & \multirow{2}{*}{$\begin{array}{c}100<V \leq \\
\pi 0\end{array}$} \\
\hline $\begin{array}{c}\text { Distancias } \\
\text { (S o S1) }\end{array}$ & s & St & S & S1 & S & S1 & s & S1 & S & S1 & S & S1 & S & S1 & & & & & \\
\hline Referencia 1 & - & - & - & 0,6 & - & 0,6 & - & 1,0 & - & 1,0 & - & 10 & - & 20 & - & 0,8 & 0,8 & 0,8 & 0,8 \\
\hline Referencia 2 & - & - & - & 1,3 & - & 1,3 & - & 1,3 & - & 20 & - & 50 & - & 15,0 & - & 1,5 & 25 & 5,0 & 7,5 \\
\hline Referencia 3 & - & - & - & 0,6 & - & 0,6 & - & 1,0 & - & 50 & - & 50 & - & 10,0 & - & 0,8 & 1,0 & 25 & 50 \\
\hline Referencia 4 & 3,0 & - & 30 & 20 & 50 & 3,0 & 7,5 & 5,0 & 10,0 & 7,5 & 150 & 10,0 & 30,0 & 20,0 & 30 & 3,0 & 40 & 50 & 100 \\
\hline Referencia 5 & 6,0 & - & 60 & - & 10,0 & - & 15,0 & - & 200 & - & 300 & - & 600 & - & 60 & 60 & 80 & 10,0 & 200 \\
\hline Referencia 6 & 3,0 & - & 3,0 & - & 3,0 & - & 3,0 & - & 30 & - & 30 & - & 3,0 & - & 3,0 & 3,0 & 30 & 3,0 & 3,0 \\
\hline \multicolumn{20}{|c|}{$\begin{array}{l}\text { S: Distancia desde válvula de alivio de presión del tanque. } \\
\text { s1: Distancia desde las paredes del tanque (s). }\end{array}$} \\
\hline \multicolumn{3}{|c|}{ Referencia 1.- } & \multicolumn{17}{|c|}{ Espacio libre alrededor de la proyección sobre el terreno del depósito. } \\
\hline \multicolumn{3}{|c|}{ Referencia 2.- } & \multicolumn{17}{|c|}{ Distancia al cerramiento de la estación. } \\
\hline \multicolumn{3}{|c|}{ Referencia 3.- } & \multicolumn{17}{|c|}{ Distancia a muros o paredes ciegas (RF-120). } \\
\hline \multicolumn{2}{|c|}{ Referencia 4.- } & \multicolumn{18}{|c|}{$\begin{array}{l}\text { Distancias al lindero de la propiedad, aberturas de inmuebles, focos fijos de inflamación, motores de } \\
\text { explosión, vías públicas, férreas o fluviales, proyección de líneas aéreas de alta tensión, equipos eléctricos } \\
\text { no protegidos, sótanos, alcantarillas y desagües. }\end{array}$} \\
\hline Referenc & ia 5 - & & $\begin{array}{l}\text { Distan } \\
\text { espect } \\
\text { Estacic }\end{array}$ & $\begin{array}{l}\text { ías } \\
\text { áculo } \\
\text { nes }\end{array}$ & $\begin{array}{l}\text { aber } \\
\text { acuar } \\
\text { Serv }\end{array}$ & $\begin{array}{l}\text { uras } \\
\text { elamie } \\
\text { cios (E }\end{array}$ & $\begin{array}{l}\text { le edi } \\
\text { tos, } \\
\text { ocas }\end{array}$ & $\begin{array}{l}\text { ificios } \\
\text { centro } \\
\text { de aln }\end{array}$ & $\begin{array}{l}\text { par } \\
\text { os cor } \\
\text { macer }\end{array}$ & $\begin{array}{l}\text { uso } \\
\text { ercial } \\
\text { amien }\end{array}$ & $\begin{array}{l}\text { doce } \\
\text { es, } \mathrm{m} \\
\text { to y p }\end{array}$ & $\begin{array}{l}\text { nte, } \\
\text { useos } \\
\text { untos }\end{array}$ & $\begin{array}{l}\text { sanita } \\
\text {, bibli } \\
\text { de di }\end{array}$ & $\begin{array}{l}\text { io, ho } \\
\text { tecas } \\
\text { tribuci }\end{array}$ & $\begin{array}{l}\text { ospeda } \\
\text { o luga } \\
\text { ión). }\end{array}$ & $\begin{array}{l}\text { e, culto } \\
\text { res de e }\end{array}$ & $\begin{array}{l}\text {, espa } \\
\text { xposici }\end{array}$ & rcimier & $\begin{array}{l}\text { nto o } \\
\text { blicos. }\end{array}$ \\
\hline Referen & cia 6. & & Dista & & boc & de ca & ga al & tanqu & ue cis & erna. & & & & & & & & & \\
\hline
\end{tabular}

Adicionalmente la Norma Técnica prevé la instalación de sistemas de protección (duchas de enfriamiento para recipientes, tomas a tierra para recipientes, sistemas de protección contra rayos, señalización y mantenimiento), los cuales minimizan la probabilidad de ocurrencia de eventos con presencia de fuego en los alrededores de los recipientes.

El presente trabajo muestra algunas de las fallas más recurrentes que se encuentran en la zona de almacenamiento de GLP en instalaciones en Ecuador, y en base a los lineamientos establecidos en la Norma Técnica Ecuatoriana INEN 2260:2010 (INEN Ecuador, 2010), se establecen algunas medidas que están tomando en cuenta los instaladores, la Autoridad Competente, las comercializadoras del combustible y los usuarios de estos sistemas para reducir el riesgo generado por el almacenamiento de este combustible.

\section{Métodos}

Se han realizado visitas a instalaciones residenciales, comerciales e industriales de GLP en el Ecuador, y se han establecido incumplimientos de estas en la seguridad en el almacenamiento de acuerdo a la Normativa vigente, sobre todo se han considerado las distancias de seguridad establecidas en la Tabla 2, y se ha verificado la existencia de mecanismos de protección, señalización y seguridad.

Por otro lado se han separado las instalaciones según el tipo de usuario: residencial, comercial o industrial; y también se muestran resultados para usuarios urbanos y rurales; y 
finalmente se verifica si los problemas de seguridad encontrados se encuentran en recipientes portátiles (cilindros) o recipientes estacionarios (tanques).

\section{Resultados y análisis de resultados}

\section{Tipos de usuarios}

La Tabla 3 muestra la cantidad de usuarios a los cuales se les han revisado sus instalaciones.

Tabla 3: Instalaciones de GLP revisadas

\begin{tabular}{lcc} 
Tipos de usuarios & Buen estado & Mal estado \\
\hline Residenciales & 64 & 3 \\
Comerciales & 75 & 37 \\
Industriales & 42 & 9 \\
Total & 181 & 49 \\
\hline
\end{tabular}

Fuente: Elaboración propia

Se toma como instalaciones en mal estado aquellas que no cumplen con los requerimientos de seguridad establecidos (distancias de seguridad hacia terceros, protecciones, señalizaciones).

La Figura 2a muestra la ubicación de las 49 instalaciones que aquejan problemas de seguridad, sean estas urbanas o rurales, y la Figura $2 \mathrm{~b}$ muestra si los problemas encontrados son en usuarios con tanques estacionarios o con cilindros.

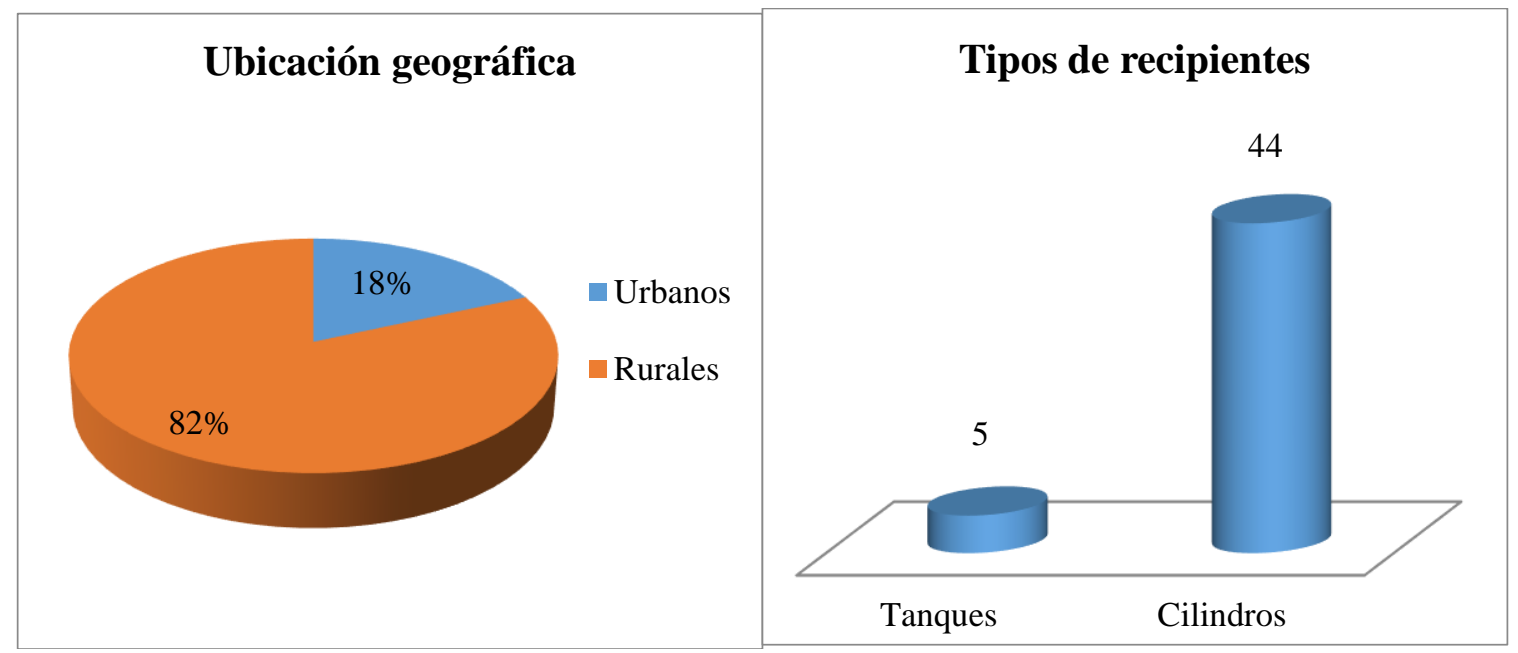

Figura 2a. Usuarios según su ubicación geográfica 2b.

Tomando en cuenta que en el Ecuador se tiene GLP con subsidio y sin subsidio (Troncoso \& Soares, 2017)(Creamer \& Becerra, 2016), hay usuarios comerciales que utilizan combustible subsidiado en sus negocios, lo cual no está permitido, por ello es comprensible encontrar mayores problemas de seguridad en este tipo de usuarios, encontrados en su mayoría en zonas rurales, utilizando cilindros para recambio. 
Para usuarios residenciales que tienen tarifa del combustible con subsidio, y para usuarios industriales, cuyos grandes consumes hacen necesaria la instalación de recipientes estacionarios, los cuales son revisados por la Autoridad Competente, se encuentran pocos problemas de seguridad (12 en total).

\section{Problemas de seguridad recurrentes}

En la Tabla 4 se indican cuáles son los problemas más recurrentes de seguridad encontrados. Hay que mencionar que existen usuarios que tienen más de uno de los criterios indicados, y que la columna \% expresa un valor porcentual respecto a la totalidad de usuarios que tienen sus instalaciones en mal estado (49).

Tabla 4: Problemas más recurrentes de seguridad

\begin{tabular}{lcc} 
Criterio de inseguridad & Nùmero de usuarios & $\%$ \\
\hline No cumplimiento de distancias mínimas & 16 & 32,65 \\
Recipientes rodeados de elementos combustibles & 25 & 51,02 \\
Falta de vaporización & 17 & 34,69 \\
Falta de sistemas de seguridad y protección & 14 & 28,57 \\
Accesorios de conexión en mal estado & 26 & 53,06 \\
\hline
\end{tabular}

Fuente: Elaboración propia

\section{No cumplimiento de distancias mínimas}

La Tabla 3 muestra las distancias mínimas que se deben respetar respecto a terceros que en un momento determinado pueden contribuir a incrementar un incidente con fuego, como en la Figura 3a que se muestra un recipiente cerca de un extractor de grasas.

\section{Recipientes rodeados de elementos combustibles}

En la Figura $3 b$ se pueden apreciar elementos combustibles cercanos al cilindro de $45 \mathrm{Kg}$.

\section{Falta de vaporización}

Se había indicado que al interior de los recipientes el GLP se encuentra en estado líquido, y en él se produce un fenómeno de vaporización natural que sirve para el consume en los equipos. Cuando estos equipos requieren más combustible del que los recipientes les pueden proporcionar, en estos se observa formación de escarcha en sus paredes (Figura 3c).

La solución es incrementar el volumen almacenado de combustible, y hay usuarios que colocan los recipientes en tinas con agua caliente para ayudar en la vaporización (Figura 3d), lo cual obviamente no es la mejor solución.

\section{Falta de sistemas de protección y seguridad}


Dependiendo de la capacidad almacenada, la Norma Técnica recomienda la instalación de sistemas de protección como duchas de enfriamiento, sistemas de pararrayos en recipientes ubicados en terrazas, tomas a tierra para descargas de cargas eléctricas pasivas acumuladas en los recipientes, y necesariamente recomienda la ubicación de extintores de polvo químico seco en las cercanías de los recipientes. En la Figura $3 e$ se puede apreciar que estos requerimientos no siempre son cumplidos por los usuarios.

\section{Accesorios de conexión en mal estado}

Todos los accesorios tienen una determinada vida útil, la cual no siempre es respetada por los usuarios que pretenden extenderla lo más posible. En la Figura $3 \mathrm{f}$ se puede apreciar la fuga del combustible a través de la manguera de conexión al cilindro.
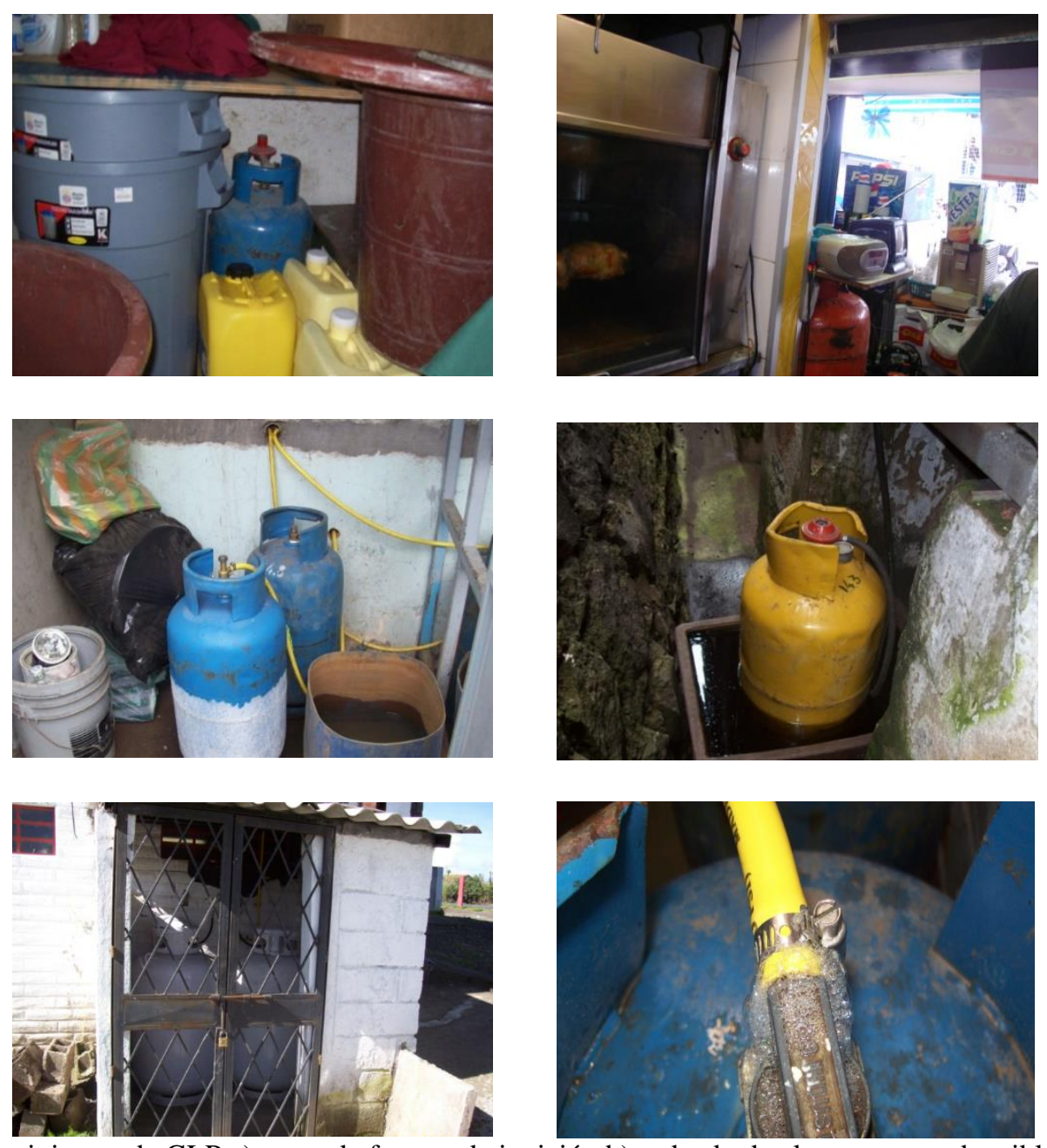

Figura 3: Recipientes de GLP a) cerca de fuentes de ignición b) rodeado de elementos combustibles c)con falta de vaporización d) ubicado en tina con agua caliente e) sin señalización ni elementos de prevención f) con accesorios en mal estado.

\section{Mejoras en las instalaciones de GLP en el Ecuador}


A partir del año 2010 donde entró en vigencia la segunda revisión a la Norma Técnica INEN 2260:2010, y como política de prevención y seguridad hacia las personas y las edificaciones, se han visto mejoras sustanciales a la seguridad de los sistemas de GLP en el Ecuador.

Hay que tener en cuenta que al tratarse de un combustible, el riesgo no puede eliminarse, pero si minimizarse (Moncada, 2015) si se toman medidas de seguridad como las contempladas en la Norma Técnica.

Las medidas más emblemáticas en la mejora de la seguridad en las instalaciones de GLP establecidas en la Norma Técnica son:

- La obligatoriedad de la instalación de sistemas de enfriamiento para recipientes ubicados en edificaciones sobre los 4 pisos (Figura 4a).

- Restricción del área de almacenamiento al acceso a personas no autorizadas a la zona de almacenamiento (Figura 4b).

- Obligatoriedad de señalizar los recipientes y su área de influencia.

- Obligatoriedad para instalar sistemas pasivos de protección en la zona de almacenamiento como descargas a tierra para cargas electrostáticas, ubicación de extintores de polvo químico seco, ubicación de pararrayos para protección de la zona de almacenamiento en edificaciones superiores a cuatro pisos (Figura 4c).

- Obligatoriedad del mantenimiento preventivo a las instalaciones que incluye las pruebas y verificaciones a los recipientes estacionarios (Figura 4d).
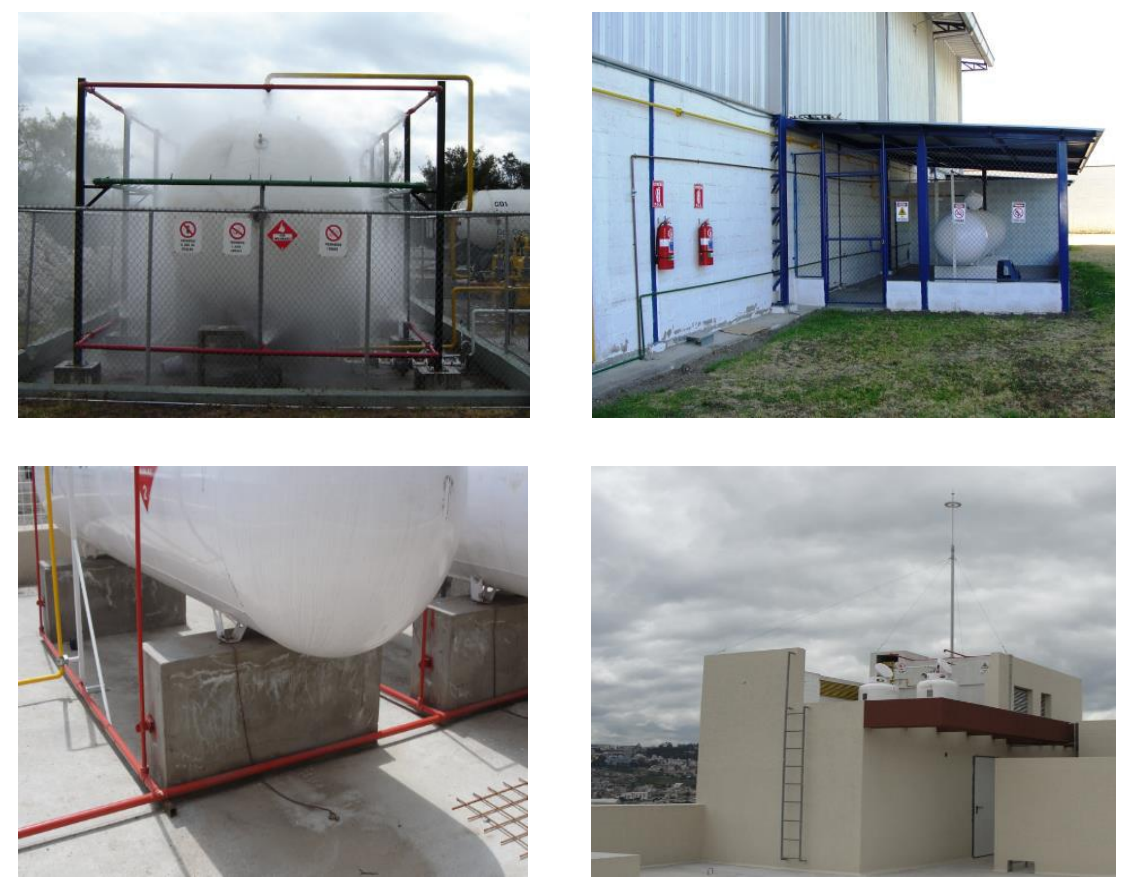

Figura 4: a) Sistema de enfriamiento sobre tanque b) Restricción al paso de extraños c) Puesta a tierra d) Pararrayos que protege el área de almacenamiento. 


\section{Conclusiones}

Sin duda alguna el almacenamiento del GLP es la parte más sensible, al momento de hacer un análisis de seguridad en un sistema que utilice este combustible.

Todo esfuerzo realizado por minimizar los riesgos y precautelar la integridad de las personas y las edificaciones vale la pena hacerlo, por ello es necesaria la inspección y el mantenimiento en las instalaciones de un combustible.

Hay que tomar en cuenta que las recomendaciones y limitaciones establecidas en Normas técnicas son las mínimas exigidas, así que lo realizado por encima de estos lineamientos en pro de la seguridad siempre va a ser bien recibido.

A partir de la segunda revisión de la Norma Técnica en 2010, y gracias al trabajo de la Autoridad Competente en Ecuador, por su trabajo de supervisión, inspección y registro, la seguridad de las instalaciones de GLP ha mejorado ostensiblemente en el país.

\section{Bibliografía}

Abbasi, T., \& Abbasi, S. A. (2007). The boiling liquid expanding vapour explosion (BLEVE): Mechanism, consequence assessment, management. Journal of Hazardous Materials, 141, 489-519. https://doi.org/10.1016/j.jhazmat.2006.09.056

Abbasi, T., \& Abbasi, S. A. (2008). The boiling liquid expanding vapour explosion (BLEVE) is fifty ... and lives on! Journal of Loss Prevention in the Process Industries, 21(4), 485-487. https://doi.org/10.1016/j.jlp.2008.02.002

Albán, H. (2016). Agencia de Regulación y Control Hidrocarburífero (ARCH) Comercialización del gas licuado de petróleo en el Ecuador. Retrieved from http://www.scpm.gob.ec/wpcontent/uploads/2014/02/2.1-Henry-Albán-ARCH-Comercializacion-de-GLP-en-elEcuador.pdf

Boggavarapu, P., Ray, B., \& Ravikrishna, R. V. (2014). Thermal Efficiency of LPG and PNG-fired burners: Experimental and numerical studies. Fuel, 116, 709-715. https://doi.org/10.1016/j.fuel.2013.08.054

Chica, F., Espinoza, F., \& Rivera, N. (2010). Gas licuado de petróleo como combustible alternativo para motores diesel con la finalidad de reducir la contaminación del aire. Ingenius Revista de Ciencia Y Tecnologia, 4, 73-81.

Creamer, B., \& Becerra, R. (2016). Cuantificación de los subsidios de derivados del petróleo a los hidrocarburos en el Ecuador. Petróleo Al Día. Boletín Estadístico Del Sector de Hidrocarburos, 2, 9-26.

Eckhoff, R. K. (2014). Boiling liquid expanding vapour explosions (BLEVEs): A brief review. Journal of Loss Prevention in the Process Industries, 32(1), 30-43. https://doi.org/10.1016/j.jlp.2014.06.008 
INEN Ecuador. (2010). NTE INEN 2260:2010 Instalaciones de gases combustibles para uso residencial, comercial e industrial. Requisitos (2da Rev.). Instituto Ecuatoriano de Normalización.

International Labour Office. (1993). Major hazard control: A practical manual (Third Edit).

Kim, J., Kim, K., \& Oh, S. (2016). An assessment of the ultra-lean combustion direct-injection LPG (liquefied petroleum gas) engine for passenger-car applications under the FTP-75 mode. Fuel Processing Technology, 154, 219-226. https://doi.org/10.1016/j.fuproc.2016.08.036

Kities, R., Mulder, P., \& Rietveld, P. (2014). Energy poverty reduction by fuel switching. Impact evaluation of the LPG conversion program in Indonesia. Energy Policy, 66, 436-449. https://doi.org/10.1016/j.enpol.2013.11.021

Leporini, L. (2017). Modelling the pressurization induced by solar radiation on above ground installations of LPG pipeline systems. Journal of Physics: Conference Series, 0-9. https://doi.org/923 012023

Moncada, J. (2015). Riesgo o peligro. NFPA Journal Latinoamericano, 4(2), 6-7.

Nardini, G., \& Nolasco, J. (2014). Manual de Seguridad para el Llenado de Aerosoles con Propelente Hidrocarburo. Instituto Mexicano del Aerosol.

Pérez Carmona, R. (2001). Diseño de instalaciones hidrosanitarias y de gas para edificaciones (3a Edición). Bogotá.

Planas, E., Pastor, E., Casal, J., \& Bonilla, J. M. (2015). Journal of Loss Prevention in the Process Industries Analysis of the boiling liquid expanding vapor explosion (BLEVE) of a lique fi ed natural gas road tanker: The Zarzalico accident. Journal of Loss Prevention in the Process Industries, 34, 127-138. https://doi.org/10.1016/j.jlp.2015.01.026

Prugh, R. (1991). Quantify BLEVE hazards. Journal of Fire Protection Engeeniering Chem. Eng. Prog., 66-72.

Raslavi, L., Mockus, S., Ker, N., \& Starevi, M. (2014). Liquefied petroleum gas (LPG) as a medium-term option in the transition to sustainable fuels and transport. Renewable and Sustainable Energy ReviewsReviews, 32, 513-525. https://doi.org/10.1016/j.rser.2014.01.052

Shariff, A. M., Wahab, N. A., \& Rusli, R. (2016). Assessing the hazards from a BLEVE and minimizing its impacts using the inherent safety concept. Journal of Loss Prevention in the Process Industries, 41, 303-314. https://doi.org/10.1016/j.jlp.2016.01.001

Troncoso, K., \& Soares, A. (2017). LPG fuel subsidies in Latin America and the use of solid fuels 
to cook. Energy Policy, 107(January), 188-196. https://doi.org/10.1016/j.enpol.2017.04.046

Venegas, D. (2016). Seguridad en la instalacion de gases industriales (GLP). In II Congreso Internacional de Energía República Dominicana (p. 14).

Venegas, D., \& Ayabaca, C. (2017). Instalaciones de gas licuado de petróleo. (Editorial Académica Española, Ed.) (First Edit).

Venegas, D., \& Farías, Ó. (2017). La BLEVE, un motivo para la seguridad en las instalaciones de GLP. In 13o Congreso Iberoamericano de Ingeniería Mecánica CIBEM. Lisboa.

Venegas, D., Yanez, J., Celi, S., Ayabaca, C., Tipanluisa, L., Bastidas, D., \& Arrocha, M. (2016a). Mantenimiento necesario en instalaciones de GLP. In Asociacion Española de Ingenieria Mecanica (pp. 353-360). Elche.

Venegas, D., Yanez, J., Celi, S., Ayabaca, C., Tipanluisa, L., Bastidas, D., \& Arrocha, M. (2016b). Mantenimiento necesario en instalaciones de GLP. In Asociacion Española de Ingenieria Mecanica. Elche.

Venegas Vásconez, D., Arrocha Carrera, M., Celi Ortega, S., Rocha Hoyos, J., Ayabaca Sarria, C., \& Mena Mena, E. (2017). Manejo inseguro del gas licuado de petróleo en Panamá. RIDTEC I+D Tecnológico, 13(2), 22-30. 